\title{
Caracterisation des Services Ecosystemiques dans la Reserve de Biosphere Transfrontaliere du W (RBTW) au Nord-Benin
}

\author{
Bernadette Sabi Lolo Ilou, \\ Ismaïla Toko Imorou,
}

Laboratoire de Cartographie (LaCarto), Université d'Abomey-Calavi (UAC)

Toussaint Vigninou,

Laboratoire d'Etudes des Dynamiques Urbaines et Régionales (LEDUR),

Université d'Abomey-Calavi (UAC)

\section{Omer Thoma,}

Laboratoire de Cartographie (LaCarto), Université d'Abomey-Calavi (UAC)

Doi:10.19044/esj.2019.v15n36p278 URL:http://dx.doi.org/10.19044/esj.2019.v15n36p278

\section{Résumé}

La Réserve de Biosphère Transfrontalière du W fournie d'importants services aux populations riveraines. Les services écosystémiques sont les bénéfices que les êtres humains tirent des écosystèmes. Cette notion permet de mieux comprendre l'interdépendance entre les écosystèmes et les sociétés humaines. Le but de cette recherche est de caractériser les services écosystémiques dans la Réserve de Biosphère Transfrontalière du W (RBTW) suivant les villages, les groupes socioculturels, les groupes socioprofessionnels et le genre. La formule de Schwartz a été utilisée pour déterminer la taille de l'échantillon des groupes socioculturels et socioprofessionnels. La caractérisation des services écosystémiques a été faite à partir de l'Analyse Factorielle de Correspondance et de la Classification Hiérarchiques Ascendante (CHA). Dans la RBTW, 237 services écosystémiques ont été identifiés. Les 32 services écosystémiques les plus sollicités ont été regroupés en 11 catégories notamment les PFNL, les produits médicinaux, les bois, les produits agricoles et les produits de chasse. La CHA a conduit à la répartition des villages en quatre groupes. La caractérisation des services écosystémiques dans la RBTW a montré que les services d'approvisionnement sont les plus exploités. L'utilisation des services écosystémiques ne dépend pas seulement des groupes socioculturels et socioprofessionnels mais aussi du genre. La commercialisation des produits issus des services écosystémiques de la RBTW contribue efficacement à l'augmentation des revenus annuels des ménages. 
Mots clés: Services Écosystémiques, Diversité, Groupes Socioculturels, Utilisation, RBTW, Bénin

\title{
Characterization of Ecosystemic Services in the "W" Transboundary Biosphere Reserve (WTBR) in Northern Benin
}

\author{
Bernadette Sabi Lolo Ilou, \\ Ismaïla Toko Imorou,
}

Laboratoire de Cartographie (LaCarto), Université d'Abomey-Calavi (UAC)

Toussaint Vigninou,

Laboratoire d'Etudes des Dynamiques Urbaines et Régionales (LEDUR), Université d'Abomey-Calavi (UAC)

\section{Omer Thoma,}

Laboratoire de Cartographie (LaCarto), Université d'Abomey-Calavi (UAC)

\section{Abstract}

The W Transboundary Biosphere Reserve provides important services to local populations. Developed and widely disseminated by the Millennium Ecosystem Assessment, ecosystem services are the benefits that humans derive from ecosystems. This notion provides a better understanding of the interdependence between ecosystems and human societies. The aims of this research is to characterize ecosystem services in the W Transboundary Biosphere Reserve (WTBR) according to villages, socio-cultural and socioprofessional groups, gender. Schwartz's formula was used to determine the sample size of sociocultural and socio-professional groups. The characterization of ecosystem services was made from Correspondence Factor Analysis and Hierarchical Ascending Classification (CHA). In the WTBR, 237 ecosystem services have been identified. The 32 most used ecosystemic services were grouped into 11 categories including NTFPs, medicinal products, woods, agricultural products and hunting products. CHA has led to the distribution of villages into four groups. The characterization of ecosystem services in the RBTW has shown that supply services are the most exploited. The use of ecosystem services does not only depend on socio-cultural and socio-professional groups, but also on gender. The commercialization of 
WTBR's ecosystem services products effectively contributes to the increase in household annual incomes.

Keywords: Ecosystem Services, Diversity, Socio-Cultural Groups, Use, RBTW, Benin

\section{Introduction}

Les services écosystémiques sont des avantages tirés des ressources naturelles par les communautés. Ils sont cruciaux pour la survie et le développement des sociétés humaines. Le concept de service écosystémique est devenu le modèle incontournable du lien entre le fonctionnement des écosystèmes et le bien-être humain (Fisher et al., 2009). Le développement de la notion de service écosystémique n'est pas un phénomène nouveau. En Afrique et au Bénin, plusieurs études ont porté sur l'évaluation des services écosystémiques. On peut citer, entre autre, les travaux de Gnangle (2017), Sabi Lolo et al. (2017), Diatta et al. (2016), Dieng et al. (2016), Mensah et al. (2016), Sabi Lolo (2015), Bene et al. (2015), Baba Cheick (2013), Daniel et al. (2012), Malan Garba (2009). Ces différentes études ont abordé la diversité, l'utilité et la contribution des services écosystémiques aux revenus des ménages d'une part, la séquestration du carbone et l'impact des feux de végétation sur les services écosystémiques d'autre part. Dans la Réserve de Biosphère Transfrontalière du W (RBTW), plusieurs études sont menées sur l'évaluation de la biodiversité et la dégradation des ressources naturelles ; mais peu ont abordé le concept de services écosystémiques. Or, les services rendus par les aires protégées en générale et la RBTW en particulier peuvent être classées parmi les quatre catégories de services écosystémiques définies par le MEA (2005). Véritable support de biodiversité, la RBTW est une source fondamentale de ressources alimentaires et médicinales pour les communautés riveraines et les animaux (services d'approvisionnement). Elle participe, à travers la diversité de ses écosystèmes, au maintien d'une flore et d'une faune sauvage diversifiée et de leurs habitats (service de soutien). La RBTW joue un rôle dans la régulation du climat par la fixation et le stockage du carbone (service de régulation). Enfin, grâce à ses sites naturels (chute de Koudou, rapides de Barou) et ses sites sacrés (Kouta gando, Kouta kaba, Gando), la RBTW confère une valeur culturelle, cultuelle et esthétique appréciée par tous (service culturel).

Malgré la diversité des services écosystémiques dans la RBTW et leurs importances tant pour les communautés riveraines que pour les animaux, cette réserve subit des dégradations d'ordres naturel et anthropique. Si aucune mesure hardie n'est prise, la RBTW risque de perdre tout son patrimoine. Dans une région qualifiée de bassin cotonnier, la RBTW apparaît comme un îlot de verdure, un véritable refuge pour les derniers grands fauves du département. 
Ainsi, pour attirer l'attention des différents acteurs sur l'importance, la valeur économique et le devenir des services écosystémiques dans la RBTW, il urge alors de caractériser ces services.

\section{Cadre d'étude}

Le cadre d'étude se localise dans le département de l'Alibori au NordBénin. Il est situé entre $11^{\circ} 10^{\prime}$ et $12^{\circ} 23^{\prime}$ de latitude nord et entre $1^{\circ} 60^{\prime}$ et $3^{\circ} 13^{\prime}$ de longitude est. Il prend en compte 24 villages administratifs répartis dans 11 arrondissements et quatre communes (Figure 1).

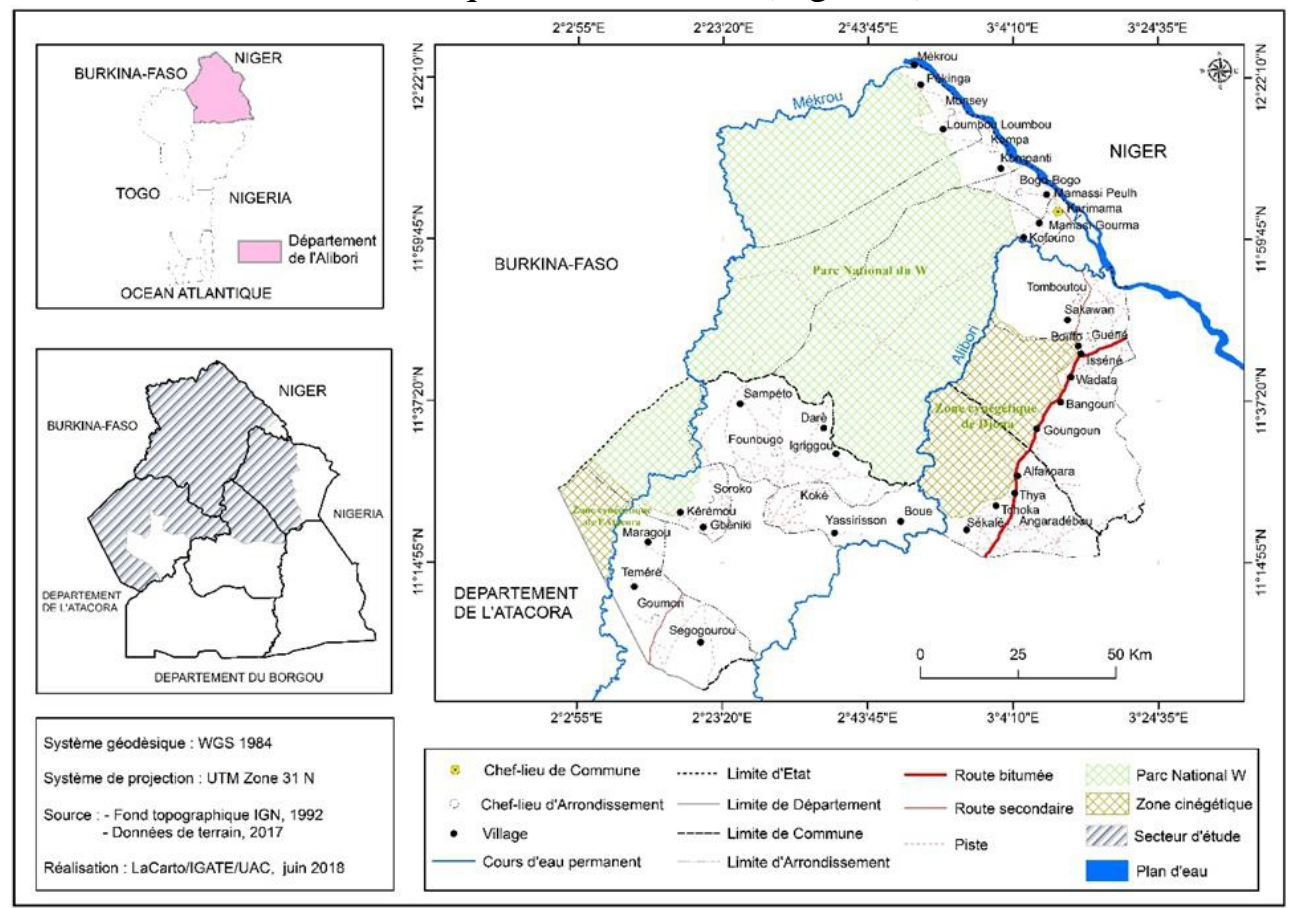

Figure 1 : Localisation du cadre d'étude

La RBTW bénéficie d'un climat de type soudanien caractérisé par deux saisons contrastées avec une pluviosité moyenne annuelle comprise entre $850 \mathrm{~mm}$ et $1000 \mathrm{~mm}$ (ASECNA, 2016). Le cumul de 1'ETP pour les 6 mois de la saison sèche est égal à $861,76 \mathrm{~mm}$ et représente $52,80 \%$ du total annuel (ASECNA, 2016). La température moyenne mensuelle tourne autour de 28,10 ${ }^{\circ} \mathrm{C}$ avec une valeur maximale de $38,75^{\circ} \mathrm{C}$ (avril) et une valeur minimale de $16,21{ }^{\circ} \mathrm{C}$ (décembre). Le secteur d'étude est influencé par deux masses d'air au cours de l'année : l'alizé maritime et l'alizé continental encore appelé harmattan. La période d'harmattan coïncide avec la récolte du coton, de la paille, de certains PFNL et du passage des feux de végétation. Les principaux cours d'eau de la RBTW sont le Mékrou $(480 \mathrm{~km})$, l'Alibori $(338 \mathrm{~km})$ et leurs 
différents affluents notamment Kpako, Kompa Gorou, Kokodiangou, Bédarou, Djiga, Konékoga, Poumon, etc.

Les espèces floristiques fréquemment utilisées par les populations riveraines de la RBTW sont Adansonia digitata Sclerocarya birrea, Lannea microcarpa, Vitellaria paradoxa Parkia biglobosa et enfin Faidherbia albida. Toutes ces espèces offrent des services écosystémiques de quantité et de qualité. .)

La faune de la RBTW est composée de presque toutes les espèces des savanes soudaniennes d'Afrique occidentale à l'exception de la girafe et des élans de derby. (Programme Parc W ECOPAS, 2005). L'abondance et la diversité de cette faune sont les principales causes du braconnage dans la RBTW.

La population riveraine de la RBTW est passée de 153580 habitants en 2002 (INSAE/RGPH 3, 2002) à 441623 habitants en 2012 (INSAE/RGPH $4,2013)$. Les populations autochtones sont en majorité des Baatombou (34\%), des Peulh (21\%), des Dendi (20\%), des Mokolé (6\%) (INSAE/RGPH 4, 2013). Ils sont agriculteurs. L'effectif total des ménages agricoles est de 83475 (INSAE/RGPH 4, 2013) soit $78 \%$ de la population des terroirs riverains.. La chasse est pratiquée dans les terroirs villageois et zones cynégétiques. La coupe de paille est l'une des récentes activités autorisées dans l'aire protégée par les autorités après la délivrance des permis de coupes. Elle constitue l'une des principales sources de revenus des paysans.

\section{Méthodes}

\subsection{Echantillonnage}

Sur la base des critères tels que localités riveraines de la RBTW, groupes socioculturels, groupes socioprofessionnels et accessibilité relative des villages, 24 villages administratifs répartis dans 11 arrondissements ont été choisis dans les quatre communes du département de l'Alibori, riveraines de la RBTW.

La formule de Schwartz (1995) a été utilisée pour déterminer la taille de l'échantillon des groupes cibles. Sur la base de cette formule, 475 personnes composées de 125 Dendi, 120 Baatombou, 95 Peulh, 82 Mokolé et 53 Gourmantché ont été interrogées.

Selon l'importance des groupes socioprofessionnels et socioculturels, deux types de choix ont été opérés : le choix raisonné et le choix aléatoire. Pour les groupes socioprofessionnels et socioculturels ayant un effectif limité, le choix a été raisonné. Par contre, le choix a été aléatoire pour les groupes socioprofessionnels et socioculturels majoritaires. La technique de boule de neige qui consiste à interroger les acteurs à tour de rôle l'un référent l'autre jusqu'à saturation a été utilisée. 


\subsection{Collecte des données}

Les enquêtes socioéconomiques et socio-anthropologiques et les observations directes ont permis de caractériser les services écosystémiques de la RBTW. Les enquêtes ont été menées suivant l'approche d'Analyse Socioéconomique selon le Genre (ASEG), basée sur l'âge, le sexe et les marginalisés des groupes socioculturels et socioprofessionnels.

Des entretiens structurés ont été réalisés à l'aide de questionnaires auprès des ménages dans les terroirs villageois. Un questionnaire a été administré individuellement aux différentes catégories des populations. Cette technique d'entretien individuel a été complétée par un focus group dans chaque village. La matrice d'orientation stratégique (MOS) a permis aux populations de déterminer les services écosystémiques les plus importants au cours des focus group. Les principaux aspects abordés dans les questionnaires sont : les caractéristiques des systèmes de productions, les modes d'utilisation, la complexité et la dynamique des services écosystémiques.

Des entretiens semi-structurés (individuels et de groupe) ont été réalisés avec des personnes ressources (chefs traditionnel, chefs religieux, etc.) sur la base d'un guide d'entretien afin de mieux connaître les formes d'utilisation des services écosystémiques par les populations.

Un guide d'entretien a été administré aux autorités politicoadministratives, aux gestionnaires du Parc W, aux éco-gardes et aux membres des AVIGREF.

Enfin, des observations participantes à travers une grille d'observation ont permis d'identifier in situ les différents services écosystémiques dans la RBTW.

\subsection{Traitement des données}

L'importance des services écosystémiques a été appréciée à travers le taux de réponse (Seastrom, 2001), la valeur d'importance et la valeur consensuelle (Byg et Balslev, 2001). Ces trois paramètres ont été utilisés pour réaliser les différents tableaux et figures.

\section{$\checkmark$ Taux de réponse}

Le taux de réponse au niveau des enquêtés par catégorie de services écosystémiques a été calculé par la formule (Seastrom, 2001) suivante :

\section{$\mathrm{T}=\mathbf{n s} / \boldsymbol{N} \times \mathbf{1 0 0}$}

$\mathrm{T}$ : taux de réponse des enquêtés (\%);

ns : nombre d'enquêtés ayant fourni une réponse pour un service écosystémique $\mathrm{s}$;

$\mathrm{N}$ : nombre de personnes interrogées dans cette catégorie ;

$\mathrm{T}$ varie entre 0 et $100 \%$. Les valeurs proches de 0 indiquent que beaucoup d'enquêtés ont une connaissance très limitée d'un service écosystémique. Par 
contre, les valeurs proches de 100 indiquent que beaucoup d'enquêtés ont une bonne connaissance d'un service écosystémique.

\section{$\checkmark$ Valeur d'importance des services écosystémiques}

La valeur d'importance (IV) des services écosystémiques est la proportion d'enquêtés qui considèrent une activité comme un service écosystémique. Elle varie de 0 à 1. Elle est déterminée par la formule (Byg \& Balslev, 2001) suivante :

\section{$I V=$ nis $/ \mathbf{n}$}

nis : nombre d'enquêtés qui considèrent un service ou un bien comme un service écosystémique ;

$\mathrm{n}$ : nombre total d'enquêtés ;

IV varie entre 0 et 1 . Les valeurs proches de 0 indiquent que très peu d'enquêtés ont une bonne connaissance d'un service écosystémique. Par contre, les valeurs proches de 1 indiquent que beaucoup d'enquêtés ont une bonne connaissance d'un service écosystémique.

\section{$\checkmark$ Valeur consensuelle du choix des services écosystémiques}

La valeur consensuelle (UCs) du choix des services écosystémiques mesure le degré de concordance du choix des services écosystémiques effectué par les enquêtés. Elle est calculée par la formule (Byg et Balslev, 2001) suivante :

\section{$\mathrm{UCs}=\mathbf{2 n s} / \mathbf{n}-\mathbf{1}$}

ns : nombre d'enquêtés ayant choisi le service écosystémique s et $\mathrm{n}$ le nombre total d'enquêtés.

UCs varie entre 0 et 4 . Les valeurs proches de 0 indiquent que très peu d'enquêtés s'accordent sur le choix d'un service écosystémique. Par contre, les valeurs proches de 4 indiquent que beaucoup d'enquêtés sont d'accord sur le choix d'un service écosystémique.

Par ailleurs, l'analyse des préférences des populations aux services écosystémiques a été faite à partir d'une Analyse Factorielle de Correspondance (AFC) sur la base de différentes matrices de données constituées par les services et plusieurs variables notamment le paramètre démographique, les groupes socioculturels et socioprofessionnels.

La catégorisation des terroirs villageois sur la base des services écosystémiques extraits de la RBTW par les populations riveraines a été réalisée en utilisant successivement l'Analyse Factorielle de Correspondance (AFC) et la Classification Hiérarchiques Ascendante (CHA). La Classification Hiérarchiques Ascendante (CHA) a été faite sur les axes factoriels de l'AFC dans le but de former des groupes homogènes de terroirs villageois.

Les différentes analyses statistiques ont été faites dans le logiciel R 3.4.3 (R Development Core Team, 2017). 


\section{Résultats}

La caractérisation des services écosystémiques dans la RBTW a concerné les quatre catégories de services écosystémiques : les services d'approvisionnement, culturels, de régulation et de soutien. Elle a été faite suivant les villages, les groupes socioculturels, les groupes socioprofessionnels et le genre.

\subsection{Diversité des services écosystémiques dans la RBTW}

Dans la RBTW, 237 services écosystémiques (SE) ont été identifiés parmi lesquels, 32 sont les plus sollicités. Ces 32 services écosystémiques ont été regroupés en 11 catégories (Figure 2).

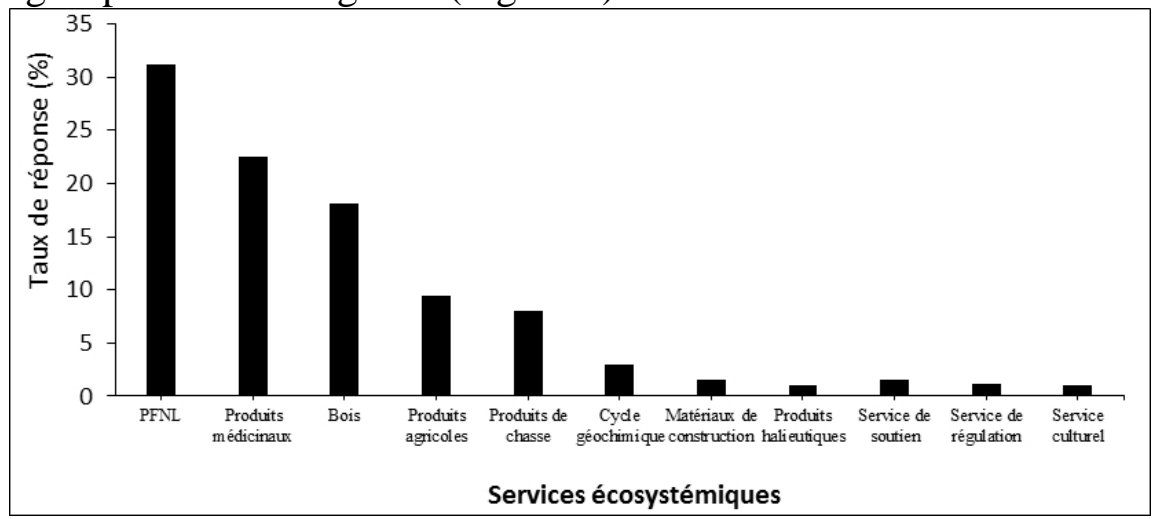

Figure 2: Catégories de services écosystémiques tirés dans la RBTW

Il ressort de l'analyse de la figure 2 que les produits forestiers non ligneux (PFNL) (31\%), suivis des produits médicinaux $(22,5 \%)$ et du bois de feu $(18,2 \%)$ sont les plus sollicités dans la RBTW. Les PFNL regroupent les fruits de Tamarindus indica, Adansonia digitata, Vitellaria paradoxa, Parkia biglobosa, Borassus aethiopum, Vitex doniana, Lannea microcarpa, Detarium microcarpum, Balanites aegyptiaca, Ximenia americana, Opilia amentacea, Diospyros mespiliformis, Hexalobus monopetalus, Moringa oleifera et Sarcocephalus latifolius; les légumes-feuilles notamment les feuilles de Adansonia digitata, Vitex doniana, Moringa oleifera, Vernonia colorata, Vernonia cinerea, Vernonia amigdalina, Ceiba pentandra, Guiera senegalensis, Vitex simplicifolia, Cassia tora et Sesamum indicum; les légumes-fleurs de Bombax costatum ; les feuilles fourragères; les champignons ; le miel et la paille.

Quant aux produits médicinaux, ils sont d'origine végétale (racines, écorces, feuilles, fruits, gommes, épines, etc.) et animale (squelettes, cornes, crottes, peaux, mues, graisses, sécrétions : bile, venin, urine, etc.).

Concernant le bois de feu, il intervient dans la production de l'énergie domestique, et est constitué des espèces ayant un pouvoir calorifique élevé 
(Burkea africana, Vitellaria paradoxa, Detarium microcarpa, Terminalia avicennioides).

Par ailleurs, les produits agricoles $(9,41 \%)$ sont issus des spéculations cultivées dans les terroirs villageois ou dans la zone tampon du Parc W. Les produits de chasse $(7,99 \%)$ sont composés de la viande boucanée, des œufs (pintades, francolins, python de séba et tortues), de différents oiseaux (francolins et pintades).

Le cycle géochimique (eau), les matériaux de construction (sable, argile et gravier), les produits halieutiques (huîtres, poissons et mollusques), les services culturels, de régulation et de soutien contribuent chacun pour moins de $5 \%$.

\subsection{Caractérisation des services écosystémiques suivant les terroirs villageois}

Les villages riverains de la RBTW ont été caractérisés selon l'utilisation des services écosystémiques. Le dendrogramme de la classification hiérarchique ascendante des villages sur la base des services écosystémiques a donnée quatre groupes de villages (Figure 3).

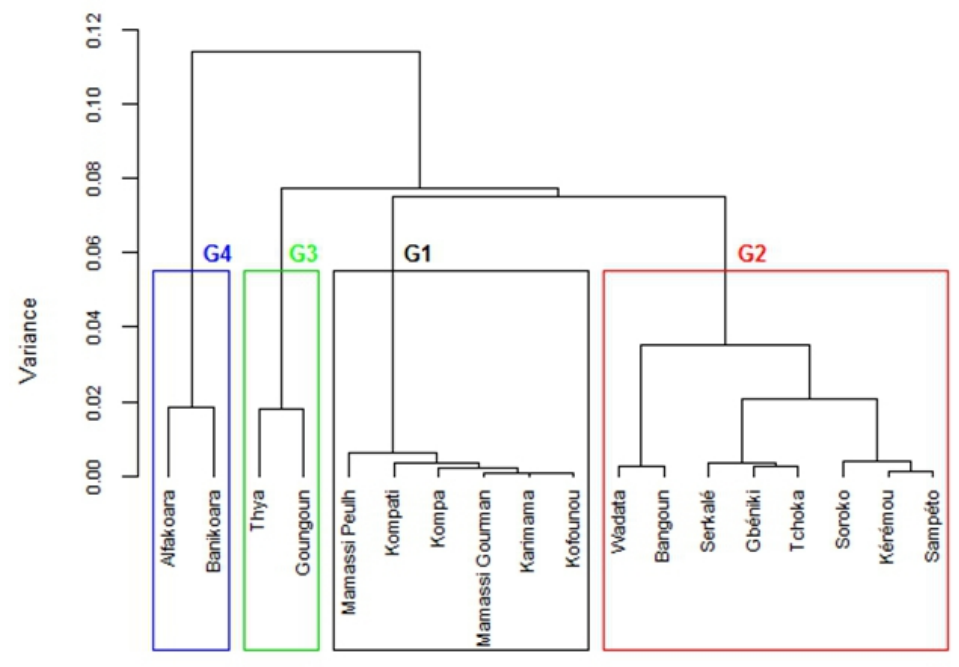

Figure 3 : Dendrogramme de la classification hiérarchique ascendante des villages

La classification hiérarchique a conduit à la répartition des villages en quatre groupes (figure 3). Le groupe 1 est composé des villages à forte exploitation des éléments du cycle géochimique, les racines, les habitats naturels, les sites rituels et l'eau des sources. Le groupe 2 est composé de villages dans lesquels la population exploite fortement les bois de feu et de charpente, la paille et les cailloux. Quant au groupe 3, il est composé des villages dans lesquels les services comme l'argile, l'éducation, les feuilles et la protection sont les plus exploités. Le groupe 4 est composé des villages dans 
lesquels les services tels que les crottes, les peaux, les cornes et la viande boucanée sont les plus utilisés.

La projection de ces quatre groupes de villages sur le plan formé par les axes factoriels 1 et 2 est présentée sur la figure 4 .

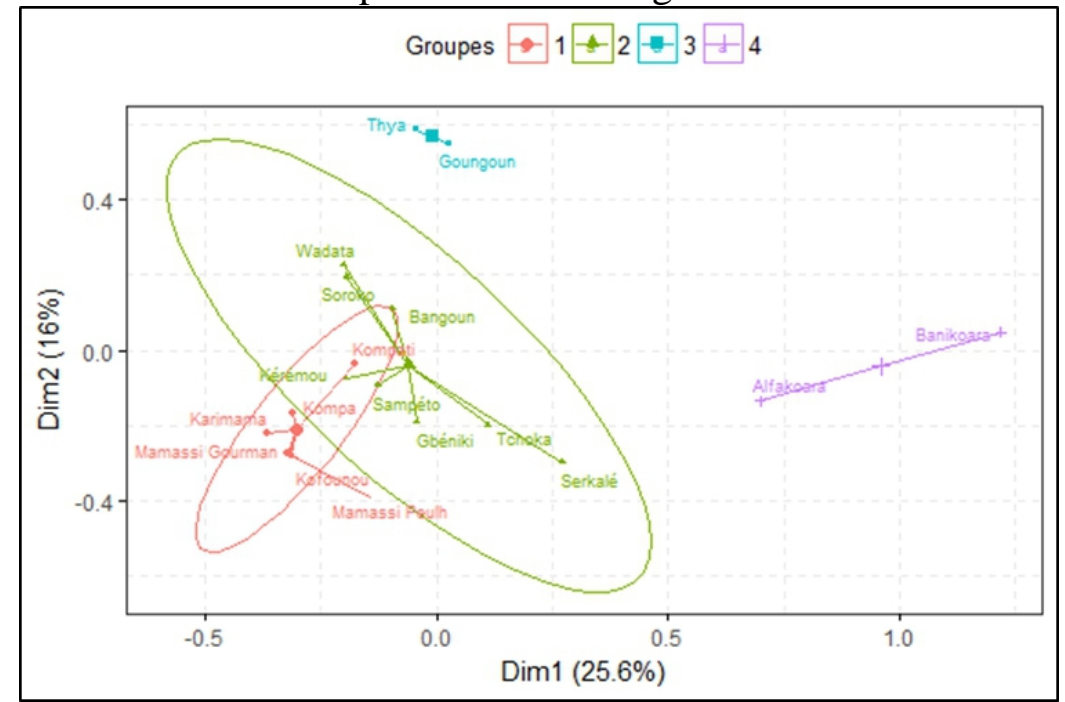

Figure 4 : Projection des quatre groupes de villages dans le plan factoriel 1 x 2

L'analyse factorielle montre que cinq axes factoriels contrôlent 73,08 $\%$ de la variance commune dont $41,6 \%$ pour les deux premiers axes (Figure 4). Les services qui ont une forte contribution dans la formation de l'axe factoriel 1 sont les crottes, les peaux, les mollusques, les cornes, les squelettes et la viande boucanée. Sur l'axe factoriel 2, les services tels que l'argile, l'éducation, les feuilles, la protection et l'eau des sources présentent les plus fortes contributions. Le groupe 1 est composé des villages à forte exploitation des éléments du cycle géochimique, les racines, les habitats naturels, les sites rituels et l'eau. Le groupe 2 est composé de villages dans lesquels la population exploite fortement les bois, la paille et les cailloux.

\subsection{Caractérisation des services écosystémiques suivant les groupes socioculturels}

La caractérisation des services écosystémiques suivant les groupes socioculturels présents dans les terroirs riverains de la RBTW est présentée sur la figure 5 . 


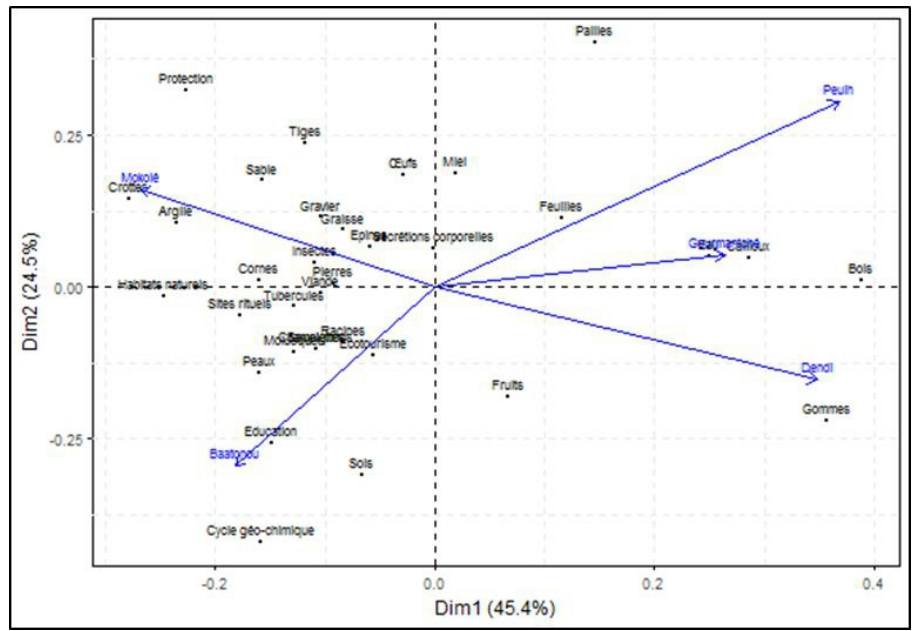

Figure 5 : Services écosystémiques selon les groupes socioculturels riverains de la RBTW

La prise en compte des deux premiers axes factoriels a permis de mettre en évidence $69,9 \%$ des variations dans l'analyse de la préférence des groupes socioculturels pour certains services écosystémiques de la RBTW (Figure 5). La projection des groupes socioculturels sur le plan formé par les axes factoriels 1 et 2 a permis de noter que les Mokolé exploitent beaucoup plus les matériaux de construction (argile, sable, gravier), les produits médicinaux (crottes, graisse, sécrétions, cornes, épines, pierres) et les habitats naturels. Les Dendi exploitent en majorité les bois de feu et de charpente, les gommes végétales et les fruits. Quant aux Baatombou, ils s'intéressent plus aux éléments du cycle géochimique, aux services éducatifs et écotouristiques, aux racines, aux peaux et aux mollusques. Les services les plus exploités par les Peulh sont les feuilles et la paille. Concernant les Gourmantché, ils s'intéressent plus aux services relatifs aux eaux de sources et aux cailloux.

\subsection{Caractérisation des services écosystémiques suivant les groupes socioprofessionnels}

La caractérisation des services écosystémiques suivant les groupes socioprofessionnels présents dans les terroirs riverains de la RBTW est présentée sur la figure 6. 


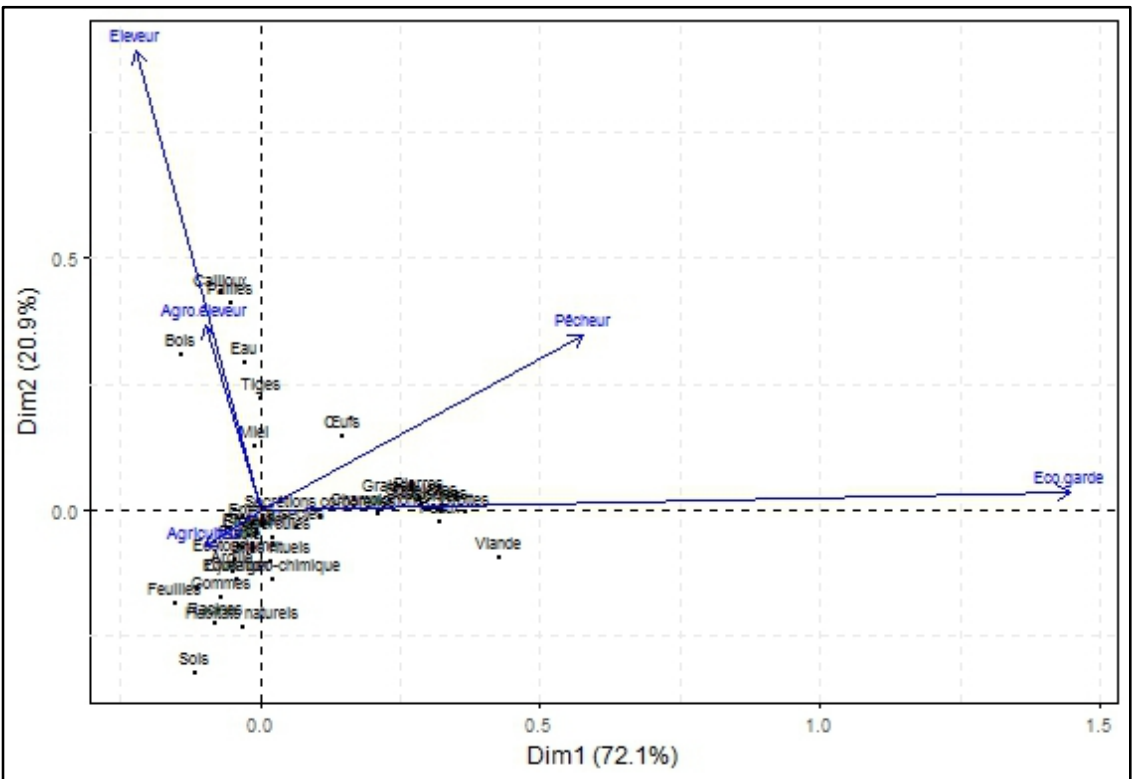

Figure 6: Services écosystémiques selon les groupes socioprofessionnels dans la RBTW

De l'analyse factorielle des interactions entre les groupes socioprofessionnels et les services écosystémiques, les deux premiers axes factoriels expliquent $93 \%$ de la variance contenue dans les données initiales (Figure 6). Le positionnement des groupes socioprofessionnels sur les axes factoriels a permis de noter que les éco-gardes ont une grande préférence pour les cornes, les crottes, les peaux, la viande boucanée, les squelettes, la graisse, les champignons, les mollusques et les pierres. Quant aux éleveurs, ils exploitent plus les pailles, les tiges, le miel et les eaux de source alors que la préférence des agriculteurs est orientée vers les racines, les produits agricoles, les sols, le cycle géochimique, les gommes et les habitats naturels. Les agroéleveurs s'intéressent plus aux bois de feu et de charpente et aux tubercules tandis que les pêcheurs exploitent plus les œufs et les sécrétions corporelles (Figure 6).

\subsection{Caractérisation des services écosystémiques selon le genre}

La caractérisation des services écosystémiques selon le genre est présentée sur la figure 7. 


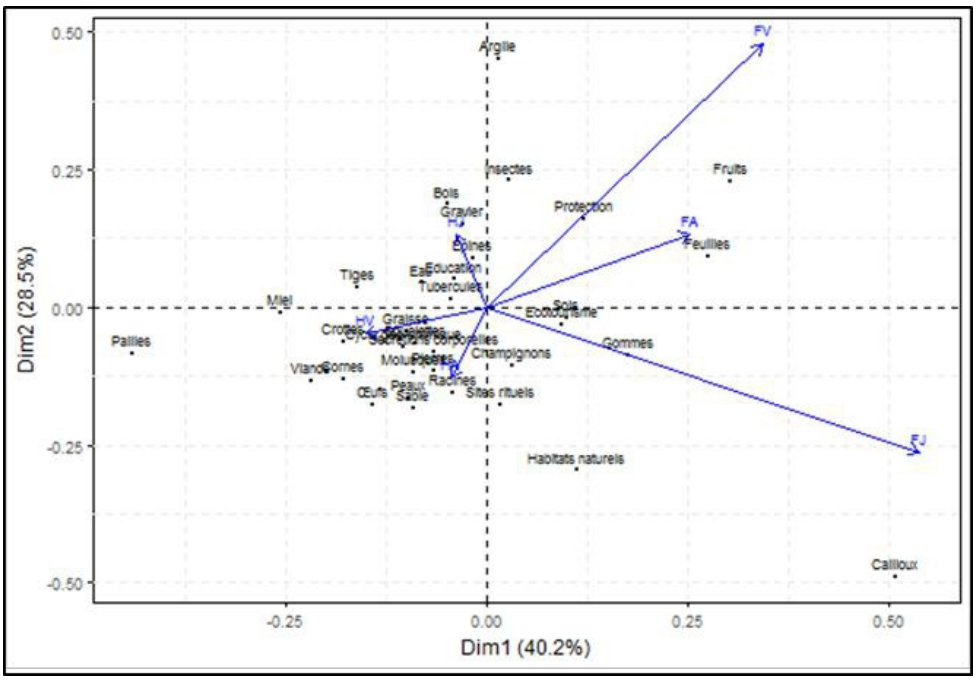

Figure 7 : Services écosystémiques selon le genre dans la RBTW

L'analyse factorielle a montré que les deux premiers axes factoriels expliquent $68,7 \%$ de la variance contenue dans la matrice de données pour mieux analyser les interactions entre le genre et les services écosystémiques tirés de la réserve (Figure 7). La projection des modalités du genre sur le plan factoriel formé par les axes 1 et 2 a révélé que les jeunes femmes ont une grande préférence pour l'extraction des cailloux et des gommes végétales tandis que les vielles ont une grande préférence pour l'exploitation de l'argile et des insectes. Par ailleurs, les femmes adultes ont une grande préférence pour l'exploitation des cornes, des feuilles et des fruits. Quant aux jeunes hommes, ils s'intéressent aux matériaux de construction (argile et gravier), aux insectes, aux sols et aux services éducatifs. Les hommes adultes ont une forte appréhension des habitats naturels, des sites rituels, des bois de feu et de charpente, des tubercules, des mollusques, des œufs, du sable et des pierres. Concernant les vieux, ils sont plus spécialisés dans l'exploitation des cornes, de la viande boucanée, de la paille, des tiges, du miel et des éléments du Cycle géochimique.

\section{Discussion}

Les services d'approvisionnement sont les plus diversifiés. La RBTW regorge une diversité de produits forestiers non ligneux (PFNL) (fruits à gousse, à pulpe, à baie et à noix, légumes-feuilles, légumes-fleurs, feuilles fourragères, champignons, bambous, pailles, écorces, miel), de produits médicinaux (racines, écorces, feuilles, fruits, gommes, épines, squelettes, cornes, crottes, peaux, mues, graisses, bile, venin, urine) et de produits agricoles (maïs, mil, sorgho, niébé, igname, riz, arachide et coton). Ces résultats confirment ceux obtenus au Bénin et dans la sous-région par Gnanglè 
et al. (2017), Diatta et al. (2016), Dieng et al. (2016), Mensah et al. (2016), Sabi Lolo et al. (2017), Bene et al. (2015) et Gnanglè (2005), pour qui la nature offre aux populations une multitude de services écosystémiques qui contribuent à leur bien-être.

La diversité des services écosystémiques dans la RBTW peut s'expliquer par la diversité des écosystèmes, l'hétérogénéité de la végétation, la diversité des groupes socioculturels qui ont diverses connaissances de ces services et la présence de l'aire protégée. Plusieurs auteurs notamment Djibril Abdoulaye et al. (2018) et Toko Imorou (2005) sont parvenus à la même conclusion selon laquelle la région soudanienne du Bénin présente une diversité d'espèces, d'écosystèmes et de paysages. La diversité de paysages est aussi le fruit d'un effort des populations. Cissé et al. (2018) ont démontré que dans le bassin versant de Boura, les connaissances ethnobotaniques des populations contribuent à la sauvegarde des espèces ligneuses à travers les parcs agroforestiers. Cette diversité d'espèces conservées est le fruit d'un système traditionnel d'exploitation des terres dans lequel des végétaux ligneux pérennes sont délibérément conservés en association avec les cultures (Wala et $a l ., 2005$ ). Les résultats obtenus montrent que les arbres occupent une place importante dans la satisfaction des besoins des populations.

Les populations riveraines de la RBTW utilisent les services écosystémiques à diverses fins et de différentes manières. Les résultats de la présente recherche ont révélé que les populations exploitent beaucoup plus l'eau et le bois dans la commune de Karimama. En effet avec la faible couverture du réseau hydraulique, le commerce de l'eau est une activité rentable durant la période de saison sèche. A Kandi, c'est plutôt les habitats naturels et les services magiques et les matériaux de construction qui sont les plus utilisés. Signalons que dans cette commune les activités sur la promotion de l'écotourisme ont été exécutées. Dans la commune de Banikoara, ce sont les services qui ont un rapport avec les sols qui sont les plus sollicités. Longtemps considérée comme la première commune productrice de coton, les activités relevant de la production sont très valorisés. Enfin, les cycles géochimiques et la gomme sont très sollicités dans la commune de Malanville.

La caractérisation des services écosystémiques selon les groupes socioculturels a permis de constater que la forte sollicitation des matériaux de construction (argile, sable, gravier), des produits médicinaux (crottes, graisse, sécrétions, cornes, épines, pierres) et des habitats naturels par les Mokolé a pour fondement la disponibilité de ces biens et services dans la zone cynégétique de la Djona. Les Dendi exploitent en majorité les bois de feu et de charpente, les gommes végétales. Les Dendi sont majoritairement installés dans les communes frontalières et ces produits font objet d'un commerce transfrontalier. Les Baatombou s'intéressent aux services qui ont un rapport avec les sols à cause du développement de la culture du coton dont Banikoara 
est la première commune productrice. En outre, ils utilisent aussi les racines, les peaux et les mollusques car ils existent de nombreux tradithérapeutes dans ce groupe socio-culturel. Les services les plus exploités par les Peulh sont les plantes fourragères (Afzelia africana, Pterocarpus erinaceus, Khaya senegalensis, Prosopis africana) et la paille qui sont la base de l'alimentation du leurs bétails et de la construction de leurs cases. Enfin, les Gourmantché sollicitent beaucoup plus les services relatifs aux eaux de sources et aux cailloux à cause de leurs vertus médicinales.

La caractérisation des services écosystémiques selon les groupes socioprofessionnels a permis de noter que les Eco-gardes ont une forte préférence pour les cornes, crottes, peaux, viande, mollusques, squelettes, graisse, champignons et pierres car ils connaissent la valeur culturelle de ses objets. Quant aux éleveurs, ils exploitent plus les pailles, tiges et eau dont ils se servent pour l'alimentation de leur bétail. Les producteurs ont une forte préférence pour les racines, sols et habitats naturels, les feuilles, œufs et tubercules tandis que les pêcheurs exploitent plus l'eau et bois dans la réserve. Ses résultats ont aussi permis de noter que les groupes socioculturels utilisent les différents services selon leurs professions. Ces résultats confirment ceux de Maddison (2006) qui propose que pour une évaluation, il vaut mieux considérer l'expérience plutôt que leur âge.

La caractérisation des services écosystémiques selon le genre a permis de comprendre que les hommes adultes ont une forte appréhension aux habitats naturels et sites rituels mais aussi aux bois, tubercules, mollusques. Quant aux jeunes hommes, ils s'intéressent aux argiles et insectes mais aussi aux graviers tout comme les jeunes femmes qui ont une forte préférence pour l'extraction des cailloux et l'éducation. Les vieilles ont une forte préférence pour l'exploitation de l'argile et des insectes. Les femmes adultes ont une forte préférence pour l'exploitation de cornes, feuilles, fruits. L'exploitation des fruits comme le karité, le néré et leurs dérivés servent non seulement dans l'alimentation des ménages mais aussi dans les produits cosmétiques et médicaux et l'embellissement des habitations. Les revenus issus de la vente permettent aux femmes rurales de se prendre en charge et de subvenir aux besoins de leurs ménages (alimentation, habillement, scolarisation et santé). Ces résultats corroborent ceux obtenus par Dah-Dovonon et Gnanglé (2006), Sokpon et Yabi (2006) qui ont démontré que les ménages ruraux tirent entre $36 \%$ et $46 \%$ de leurs revenus des produits du karité.

\section{Conclusion}

La classification des services écosystémiques de la RBTW a été adoptée suivant les terroirs villageois, les groupes socioculturels et socioprofessionnels, et selon le genre. Cette classification a permis de constater que les services d'approvisionnement sont le plus diversifiés et 
parmi eux, les PFNL sont les plus utilisés. La diversité des usages est fonction des professions et cela amène les populations à avoir différentes perceptions des services écosystémiques. Cette diversité d'usages des services écosystémiques de la RBTW entraîne de fortes pressions sur ceux-ci. Ces pressions génèrent des conflits nés de l'utilisation concurrente des ressources naturelles.

\section{References:}

1. Baba Cheick A. K., 2013. Economic valuation of ecosystem services around a multiple purpose dam: challenges and opportunities for PES implementation and land use management. A case study of Sakabansi dam in north Benin. Master thesis, University of Dresden, 40 p.

2. Bene A. \& Fournier A., 2015. Réflexion sur la notion de services écologiques : étude de cas à Kotoudéni (Burkina Faso). Vertigo, Paris : Avancées cinématographiques, 15 (3), 18 p.

3. Byg A. \& Balslev H., 2001. Diversity and use of palms in Zahamena, eastern Madagascar. Biodiversity and Conservation 10: 951-970.

4. Dah-dovonon, Z.J. et Gnanglè, P.C., 2006. Évaluation des potentialités de développement de la filière karité dans les départements de l'Atacora et de la Donga. GTZ/ProCGRN, 93p

5. Daniel T.C. A., Muharb A., Arnberger O., Aznar J.W., Boyd K.M.A., Chan R., Costanza T., Elmqvist C.G., Flint G.H., Gobster A., GrêtRegamey R., Lave S., Muhar M., Penker M.G., Ribe T., Schauppenlehner T., Sikor I., Soloviy M., Spierenburg K., Taczanowska J., Tame A., von der \& Dunk., 2012. Contributions of cultural services to the ecosystem services agenda, Proceedings of National Academic Science 109, 23, pp. 8812-8819.

6. Diatta A. A., Ndour N., Manga A., Sambou B., Faye C. S., Diatta L., Goudiaby A., Mbow C. \& Dieng S.D., 2016. Services écosystémiques du parc agroforestier à Cordyla pinnata (Lepr. ex A. Rich.) MilneRedh., dans le Sud du Bassin Arachidier (Sénégal) Int. J. Biol. Chem. Sci. 10(6): 2511-2525.

7. Dieng S. D., Diop M., Goudiaby A., Niang-Diop F., Faye L. C., Guiro I., Sambou S., Lykke A. M. \& Sambou B., 2016. « Caractérisation des services écosystémiques fournis par Cordyla pinnata dans la périphérie de la Forêt classée de Patako au Sénégal », VertigO - la revue électronique en sciences de l'environnement.

8. Djibril Abdoulaye G., Toko Imorou I., Thomas O., Zakari S., Aouna O., 2018. Evaluation du stock de carbone au-dessus du sol dans la zone cynégétique de la Djona au nord Bénin. Acte de la conférence internationale OSFACO, pp117-133. 
9. Fisher B., Turner R. K. \& Morling P., 2009. Defining and classifying ecosystem services for decision making. Ecological Economics, 68: 643-653.

10. Gnangle R. M., Biaou S. S. H., Gnangle P. C., Balagueman O.R., Raphiou M., Dicko A., Kouagou M., et Natta A.K., 2017 Services écosystémiques fournis par les parcs à karité et leur contribution au bien-être des populations rurales dans la commune de Savè (Centre Bénin) Annales de l'Université de Parakou Série « Sciences Naturelles et Agronomie » Parakou, Bénin 7 (1) :104-112

11. Gnangle P. C., 2005. Parcs à karité (Vitellaria paradoxa) (Gaertn. C. F.) (Sapotaceae) au Bénin:

12. Importance socio-culturelle, caractérisations morphologique, structurale et régénération naturelle. Mémoire de DEA. Aménagement et Gestion des Ressources Naturelles. UAC/FSA. 113 p.

13. INSAE/RGPH 3, 2002. Estimation de la population béninoise: données socio-économiques, Cotonou, Bénin, pp 7-9

14. INSAE/RGPH 4, 2013. Effectifs de la population des villages et quartiers de villes du Bénin. Cotonou, Bénin, pp 4-8.

15. Maddison D., 2006. The perception of and adaptation to climate change in Africa. CEEPA Discussion Paper No. 10. Centre for Environmental Economics and Policy in Africa, University of Pretoria, South Africa.

16. Malam Garba H. H. 2009. Estimation des valeurs des biens et services produits par les aires protégées : cas du Parc Régional W du Niger Mémoire de fin d'étude diplôme de master en Gestion des Aires Protégées (GAP) $102 \mathrm{p}$

17. Mensah S., Veldtman R., Assogbadjo A. E., Ham C., Glèlè Kakaï R., Seifert T., 2017. Ecosystem service importance and use vary with socio-environmental factors: A study from household-surveys in local communities of South Africa. Ecosystm service, 23 : 1-8

18. M.E.A (Millenium Ecosystem Assessment), 2005. Ecosystem and Human well-being. Synthesis, Washington DC, Ed. Island Press, 155 p.

19. Programme Régional Parc W / ECOPAS, 2005. Plan d'Aménagement et de Gestion de la Réserve de Biosphère Transfrontalière W 20062010, version finale $228 \mathrm{p}$

20. Sabi Lolo I. B., Sogbohossou E.A., Toko Imorou I., Houinato M.R.B. \& Sinsin B., 2017. Diversité et importance socio-économique des services écosystémiques dans la réserve de biosphere de la pendjari au nord-benin J. Rech. Sci. Univ. Lomé (Togo), 2017, 19 (3): 15-28

21. Sabi lolo I. Bernadette., Toko Imorou I., Vigninou T. \& Thomas O., 2018. Cartographie des conflits d'usage autour des services 
écosystemiques dans la réserve de Biosphère Transfrontalière du w au Nord-Bénin. Mélanges en hommage au Professeur Thomas O., Septembre 2018, vol.3, ISBN : 978-99919-822-8-1

22. Sokpon N., \& Yabi J.A. 2006. Gestion des systèmes de production, de commercialisation et de transformation du karité au Bénin. Rapport technique, $48 \mathrm{p}$.

23. Toko Imorou I. 2005. Productivité des pâturages de savanes en relation avec les phénomènes d'érosion naturelle des sols (Dongas) dans le Parc National du W. DEA/ FLASH/ UAC, Bénin, 88 p.

24. Toko Imorou I., Djogbenou P.C., Arouna O., Sogbossi E.S. \& Sinsin B., 2015. Effets de la taille et des régions phytogéographiques sur la diversité floristique et la structure des forêts sacrées au Bénin. Annales des Sciences Agronomiques (Bénin), 19 (1) : 79-97.

25. Wala K., Sinsin B., Guelly A. K., Kokou K. \& Akpagana K. (2005). Typologie et structure des parcs agroforestiers dans la sous-préfecture de Doufelgou (Togo). Sécheresse, 16 (3): 1-8. 